\title{
NUMERICAL ANALYSIS OF TEMPERATURE FIELD IN A DISC BRAKE AT DIFFERENT COVER ANGLE OF THE PAD
}

\author{
Piotr GRZEŚ \\ ${ }^{*}$ Faculty of Mechanical Engineering, Department of Mechanics and Applied Computer Science, Bialystok University of Technology \\ ul. Wiejska 45C, 15-351 Bialystok, Poland \\ p.grzes@pb.edu.pl
}

\begin{abstract}
In the paper an influence of the cover angle of the pad on temperature fields of the components of the disc brake is studied. A three-dimensional finite element ( $\mathrm{FE}$ ) model of the pad-disc system was developed at the condition of equal temperatures on the contacting surfaces. Calculations were carried out for a single braking process at constant deceleration assuming that the contact pressure corresponds with the cover angle of the pad so that the moment of friction is equal in each case analysed. Evolutions and distributions of temperature both for the contact surface of the pad and the disc were computed and shown.
\end{abstract}

Key words: Temperature, Disc Brake, Cover Angle Of The Pad, Finite Element Method

\section{INTRODUCTION}

High temperature of sliding components of a disc brake in contact may result in deformations/cracks of a disc, degradation of a pad material, brake fluid vaporization, excessive wear of working surfaces, etc. (Ścieszka 1998; Yevtushenko and Grzes, 2010). Numerical calculations of temperature typically employ axisymmetric (Talati and Jalalifar, 2008, 2009; Yevtushenko and Grzes, 2014) or three-dimensional models of a disc brake (Adamowicz and Grzes, 2011a, 2011b, Baranowski et al. 2011, 2013). The first arrangement is used when the sliding velocity is high (uniform temperature distribution in the circumferential direction) or the coefficient of mutual overlap of the pad and the disc is close to the unity. Three-dimensional models allow to reveal non-uniformities on the rubbing path of the disc due to relative motion of the sliding components. However there are also approaches accounting for the three-dimensionality and a uniform distribution of a heat flux density on the contact surface of the disc for calculation of temperature (Ghadimi et al., 2013a) and thermal stresses (Ghadimi et al., 2013b). A review article on FEA of temperature fields in disc brakes and clutches was developed by Yevtushenko and Grzes (2010).

In general brake discs (single or multiple) made as solid, vented, drilled, divided etc., are combined with the pads covering the rubbing path of the disc(s) partly or within the entire circumference (aircrafts). The latter is used to increase the friction force at the same contact pressure. The pad also may be performed as single or multiple (e.g. mine hoist disc brake systems) friction areas per one side of the disc (Scieszka and Zolnierz, 2014). However, currently typical application of a disc brake system for a vehicle consists of two pads with a cover angle of about $60^{\circ}$ each and one solid or vented brake disc.

In this study a 3D FE contact model of a disc brake was developed to study an influence of a cover angle of a pad on temperature fields of sliding components. Seven different angles of the pad $\theta_{0}$ from $40^{\circ}$ to $160^{\circ}$ were tested for a single braking process at a constant deceleration, corresponding with operating parameters, dimensions, properties of materials of the disc brake of a typical passenger vehicle. Changes in transient distributions of temperature were calculated and compared at the established cover angles of the pad.

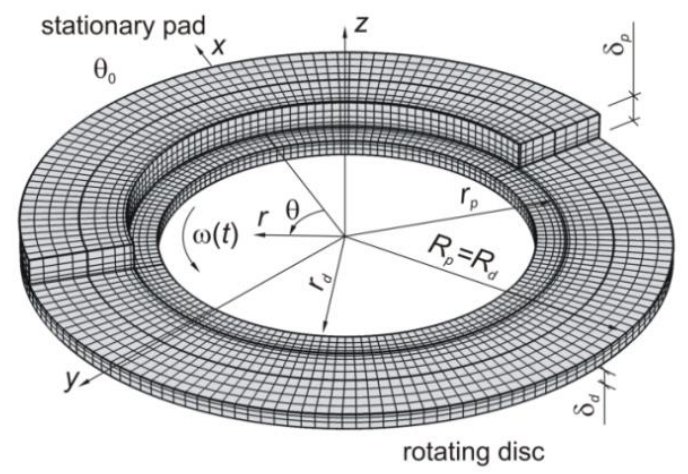

Fig. 1. 3D FE mesh of the pad-disc brake system for $\theta_{0}=160^{\circ}$

\section{BASIC EQUATIONS}

Consider a frictional heating of components of a disc brake during a single braking process of a vehicle at constant deceleration from the initial velocity $V_{0}$ to standstill according to the formula:

$V(t)=V_{0}\left(1-\frac{t}{t_{s}}\right), 0 \leq t \leq t_{s}$

where: $t$ - is time; $t_{s}$ - is the braking time.

It is assumed that the contact presure $p_{0}$ is constant and unifromly distributed on the friction surfaces of the pad and the disc. Convective cooling at the constant heat transfer coefficient takes place on the free surfaces of the pad and the disc apart from the surface/plane of symmetry of the brake disc. Thermal radiation due to short duration of the process was omitted (Adamowicz and Grzes, 2011b). 
Three-dimensional temperature fields $T_{p, d}(r, \theta, z, t)$ are calculated from the heat conduction equations for:

- the pad:

$K_{p} \Delta T=\rho_{p} c_{p} \frac{\partial T}{\partial t}, r_{p}<r<R_{p},|\theta|<0.5 \theta_{0}$

$0<z<\delta_{p}, \quad 0<t<t_{s}$

and the disc:

$K_{d} \Delta T=\rho_{d} c_{d}\left[\frac{\partial T}{\partial t}+\frac{V(t)}{R_{w}} \frac{\partial T}{\partial \theta}\right], r_{d}<r<R_{d}$

$0<\theta<2 \pi,-\delta_{d}<z<0,0<t<t_{s}$

at the following boundary conditions:

- within the contact region:

$\left.K_{d} \frac{\partial T}{\partial z}\right|_{z=0^{-}}-\left.K_{p} \frac{\partial T}{\partial z}\right|_{z=0^{+}}=q(r, \theta, 0, t)$

$(r, \theta) \in \Omega, 0 \leq t \leq t_{s}$

$T\left(r, \theta, 0^{+}, t\right)=T\left(r, \theta, 0^{-}, t\right),(r, \theta) \in \Omega, 0 \leq t \leq t_{s}$

where:

$q(r, t)=p_{0} f r R_{w}^{-1} V(t),(r, \theta) \in \Omega, 0 \leq t \leq t_{s}$

$\Delta=\frac{\partial^{2}}{\partial r^{2}}+\frac{1}{r} \frac{\partial}{\partial r}+\frac{1}{r^{2}} \frac{\partial^{2}}{\partial \theta^{2}}+\frac{\partial^{2}}{\partial z^{2}}$

$K-$ is the thermal conductivity; $c$ - is the specific heat; $\rho$ - is the density; $f-$ is the coefficient of friction; $r, R-$ is the inner and outer radius, respectively; $T$ - is temperature; $\theta_{0}$ - is the cover angle of the pad; $p_{0}$ - is the contact pressure; $R_{w}$ - is the outer radius of the wheel; $\Omega$ - is the contact area; $\delta$ - is the thickness; subscripts $p$ and $d$ denote the pad and the disc, respectively.

On the free surfaces of the pad:

$\left.\mathrm{K}_{\mathrm{p}} \frac{\partial \mathrm{T}}{\partial \mathrm{r}}\right|_{\mathrm{r}=\mathrm{r}_{\mathrm{p}}}=\mathrm{h}\left[\mathrm{T}\left(\mathrm{r}_{\mathrm{p}}, \theta, \mathrm{z}, \mathrm{t}\right)-\mathrm{T}_{\mathrm{a}}\right]$

$|\theta|<0.5 \theta_{0}, 0<\mathrm{z}<\delta_{\mathrm{p}}, 0 \leq \mathrm{t} \leq \mathrm{t}_{\mathrm{s}}$

$\left.\mathrm{K}_{\mathrm{p}} \frac{\partial \mathrm{T}}{\partial \mathrm{r}}\right|_{\mathrm{r}=\mathrm{R}_{\mathrm{p}}}=\mathrm{h}\left[\mathrm{T}_{\mathrm{a}}-\mathrm{T}\left(\mathrm{R}_{\mathrm{p}}, \theta, \mathrm{z}, \mathrm{t}\right)\right]$

$\theta<0.5 \theta 0,0<z<\delta p, 0 \leq t \leq t s$

$\left.\mathrm{K}_{\mathrm{p}} \frac{\partial \mathrm{T}}{\partial \theta}\right|_{\theta=-0.5 \theta_{0}}=\mathrm{h}\left[\mathrm{T}\left(\mathrm{r},-0.5 \theta_{0}, \mathrm{z}, \mathrm{t}\right)-\mathrm{T}_{\mathrm{a}}\right]$

$r p<r<R p, 0<z<\delta p, 0 \leq t \leq t s$

$\left.\mathrm{K}_{\mathrm{p}} \frac{\partial \mathrm{T}}{\partial \theta}\right|_{\theta=0.5 \theta_{0}}=\mathrm{h}\left[\mathrm{T}_{\mathrm{a}}-\mathrm{T}\left(\mathrm{r}, 0.5 \theta_{0}, \mathrm{z}, \mathrm{t}\right)\right]$

$r p<r<R p, 0<z<\delta p, 0 \leq t \leq t s$

$\left.\mathrm{K}_{\mathrm{p}} \frac{\partial \mathrm{T}}{\partial \mathrm{z}}\right|_{\mathrm{z}=\delta_{\mathrm{p}}}=\mathrm{h}\left[\mathrm{T}_{\mathrm{a}}-\mathrm{T}\left(\mathrm{r}, \theta, \delta_{\mathrm{p}}, \mathrm{t}\right)\right]$

$(r, \theta) \in \Omega, 0 \leq t \leq t s$

and the disc:

$\left.K_{d} \frac{\partial T}{\partial r}\right|_{r=r_{d}}=h\left[T\left(r_{d}, \theta, z, t\right)-T_{a}\right]$

$0 \leq \theta \leq 2 \pi,-\delta_{d}<z<0,0 \leq t \leq t_{s}$

$\left.K_{d} \frac{\partial T}{\partial r}\right|_{r=R_{d}}=h\left[T_{a}-T\left(R_{d}, \theta, z, t\right)\right]$

$0 \leq \theta \leq 2 \pi,-\delta_{d}<z<0,0 \leq t \leq t_{s}$
$\left.K_{d} \frac{\partial T}{\partial z}\right|_{z=0^{-}}=h\left[T_{a}-T\left(r, \theta, 0^{-}, t\right)\right]$

$r_{d}<r<r_{p}, 0 \leq \theta \leq 2 \pi, 0 \leq t \leq t_{s}$

$\left.K_{d} \frac{\partial T}{\partial z}\right|_{z=0^{-}}=h\left[T_{a}-T\left(r, \theta, 0^{-}, t\right)\right]$

$r_{p} \leq r \leq R_{p}=R_{d}, \theta \geq 0.5 \theta_{0}, 0 \leq t \leq t_{s}$

convective cooling takes place.

The surface of symmetry of the disc brake system is insulated:

$\left.\frac{\partial T}{\partial z}\right|_{z=-\delta_{d}}=0, r_{d} \leq r \leq R_{d}, 0 \leq \theta \leq 2 \pi$

$t \geq 0, z=-\delta_{d}, 0 \leq t \leq t_{s}$

Initially the pad and the disc are at ambient temperature:

$T(r, \theta, z, 0)=T_{a}, r_{p} \leq r \leq R_{p}$

$|\theta| \leq 0.5 \theta_{0}, 0 \leq z \leq \delta_{p}$

$T(r, \theta, z, 0)=T_{a}, r_{d} \leq r \leq R_{d}$

$0 \leq \theta \leq 2 \pi,-\delta_{d} \leq z \leq 0$

where: $h-$ is the heat transfer coefficient, $T_{a}-$ is the ambient/ initial temperature.

\section{FE DISCRETIZATION}

A considered thermal problem of friction is solved numerically using the finite element method. At the first step the parabolic type heat conduction equations (2), (3) were discretized using the Galerkin's method:

$[C] \frac{d\{T\}}{d t}+[K]\{T\}=\{Q\}$

where: $[C]-$ is the capacitance matrix; $\{T\}-$ is the vector of temperature, $[K]-$ is the conductivity matrix, $\{Q\}-$ is the vector of applied thermal loads.

In order to vanish in equation (20) the time derivative of temperature, the finite difference method is used with the betha parameter chosen from the range $0 \div 1$ :

$\frac{1}{\Delta t}\left[\{T\}_{t+\Delta t}-\{T\}_{t}\right]=(1-\beta)\left\{\frac{d T}{d t}\right\}_{t}+\beta\left\{\frac{d T}{d t}\right\}_{t+\Delta t}$

Substituting Eq. (21) to Eq. (20) we obtain the following set of algebraic equations:

$[[C]+\beta \Delta t[K]]\{T\}_{t+\Delta t}=[[C]-(1-\beta)[K] \Delta t]\{T\}_{t}$ $+(1-\beta) \Delta t\{Q\}_{t}+\beta \Delta t\{Q\}_{t+\Delta t}$

Computations of temperature in the pad and the disc were carried out using Comsol Multiphysics 4.4 (2013). Based on the geometrical $3 \mathrm{D}$ model, the FE mesh was constructed using hex type elements (Fig. 1). A simple geometry of the model allowed to use regular mesh. At the first step, the quad type elements on the contact surface with different distribution in radial direction were built. Then, the plane elements were swept in axial direction for the pad and the disc, respectively. A non-uniform distribution of the elements dimensions applied in the model corresponded with the expected high temperature gradient both in axial and radial directions. 


\section{NUMERICAL ANALYSIS}

Simulation of frictional heating in a pad-disc brake system during a single braking process of a vehicle from the initial velocity $V_{0}=100 \mathrm{~km} / \mathrm{h}\left(\omega_{0}=88.464 \mathrm{~s}^{-1}\right)$ to standstill at constant deceleration $a=-7.015 \mathrm{~m} / \mathrm{s}^{2}$ using FE based software Comsol Multiphysics 4.4 (2013) was carried out. The 3D FE mesh of the model for $\theta_{0}=160^{\circ}$ is shown in Fig. 1. Initial/ambient temperature is equal $T_{a}=20^{\circ} \mathrm{C}$. Dimensions of the brake components are shown in Tab. 1. The pad is made of FC-16L and the disc is cast-iron ChNMKh (Tab. 2). The materials used are typical for the sliding components of a brake, namely the thermal conductivity of the friction material is several times lower, than the thermal conductivity of the disc. The convective heat transfer coefficient on the free surfaces of the pad and the disc is constant, independent of the velocity $h=60 \mathrm{~W} /\left(\mathrm{m}^{2} \mathrm{~K}\right)$ (Talati and Jalalifar, 2009).

The main goal of the FEA was to examine the influence of the cover angle of the pad $40^{\circ} \div 160^{\circ}$ on the temperature field of the friction pair during the single braking. In each case the work done was equal $392.1 \mathrm{~kJ}$. To maintain the same moment of friction, the pressure was changed. For the automotive application, the cover angle of the pad equals approximately $60^{\circ}$, however e.g. in aircrafts the friction surface of the pad is consistent with the friction surface of the disc (rubbing path) $360^{\circ}$.

Tab. 1. Dimensions of the pad and the disc (Talati and Jalalifar, 2009)

\begin{tabular}{|c|c|c|}
\hline dimension & pad & disc \\
\hline inner radius, $r_{p, d}[\mathrm{~m}]$ & 0.0765 & 0.066 \\
\hline outer radius, $R_{p, d}[\mathrm{~m}]$ & 0.1135 & 0.1135 \\
\hline thickness $\delta_{p, d}[\mathrm{~m}]$ & 0.01 & 0.0055 \\
\hline cover angle of the pad, $\theta_{0}[\mathrm{deg}]$ & $40,60,80,100$, & \\
& $120,140,160$ & \\
\hline
\end{tabular}

Tab. 2. Thermophysical properties of materials of the pad and the disc (Chichinadze et al., 1986)

\begin{tabular}{|c|c|c|}
\hline thermophysical properties & $\begin{array}{c}\text { FC-16L } \\
\text { (pad) }\end{array}$ & $\begin{array}{c}\text { cast iron } \\
\text { ChNMKh (disc) }\end{array}$ \\
\hline thermal conductivity, $K[\mathrm{~W} /(\mathrm{mK})]$ & 0.79 & 51 \\
\hline specific heat, $c[\mathrm{~J} /(\mathrm{kgK})]$ & 961 & 500 \\
\hline density, $\rho\left[\mathrm{kg} / \mathrm{m}^{3}\right]$ & 2500 & 7100 \\
\hline
\end{tabular}

In order to identify the location of the maxiumum temperature in the pad-disc system, the distribution of temperature on the contact surface of the disc was analysed for $\theta_{0}=40^{\circ}$ and $160^{\circ}$ (Fig. 2). As expected, the temperature field is non-uniform both within the contact region and on the free surface for these two angles. The location of maxiumum temperature appears approximately for $r=R_{d}$ and $\theta=0.5 \theta_{0}$, which results from the direction of revolution of the disc and the applied heat flux density (Eq.(6)). Noticeable is the fact that the temperature for $\theta_{0}=$ $160^{\circ}$ is almost uniform in circumferential direction whereas for $40^{\circ}$ the maxiumum temperature under the pad is higher.

Temperature distributions for $\theta_{0}=40^{\circ} \div 160^{\circ}$ on the outer edge of the constact surface of the disc at the moment of half of the braking time $(t=2 \mathrm{~s})$ are shown in Fig. 3. The main difference is the maxiumum temperature reached, higher for smaller cover angle of the pad. The temperature out of the pad area is almost equal for each $\theta_{0}$ presented. a)

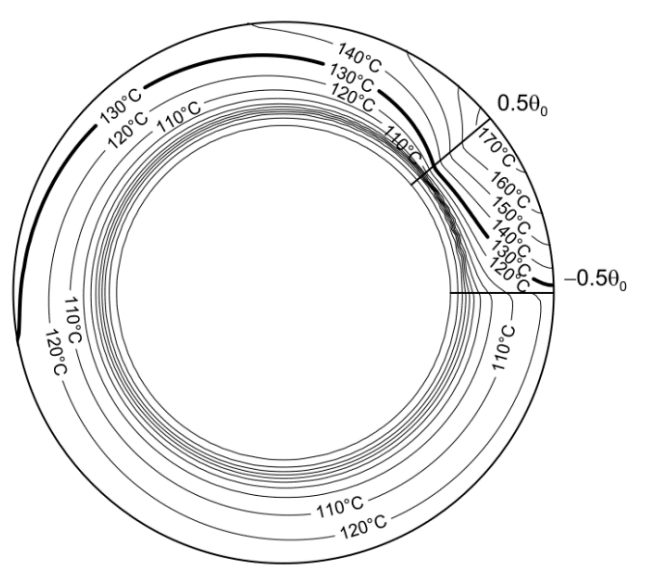

b)

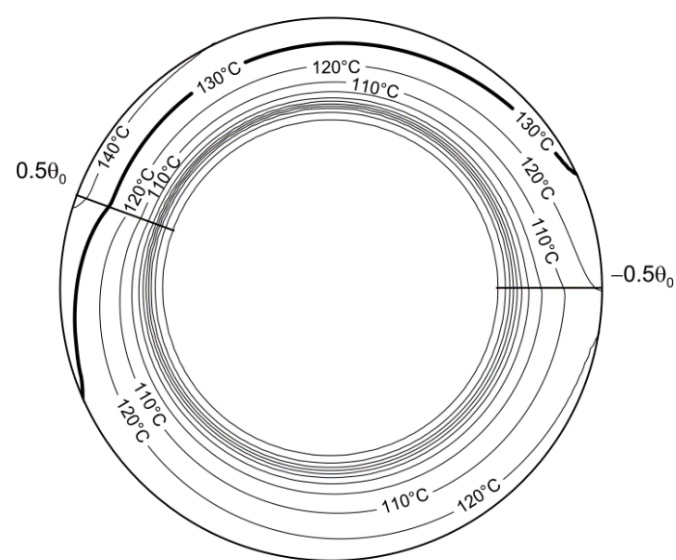

Fig. 2. Temperature distributions on the contact surface of the disc at the time moment $(t=2 \mathrm{~s})$ for the cover angle of the pad: a) $\theta_{0}=40^{\circ}$ and b) $\theta_{0}=160^{\circ}$

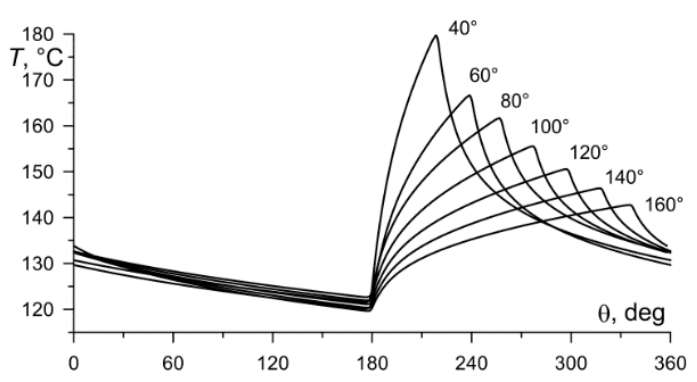

Fig. 3. Distributions of temperature in the circumeferential direction on the outer edge of the friction surface of the disc $r=R_{d}$ at different cover angles of the pad $\theta_{0}(t=2 \mathrm{~s})$

In this numerical analysis, the pad is fixed and the disc is rotating. Therefore it is convenient to analyse the temperature in the stationary region of the pad. The evolutions of the maximum temperature on the contact surface of the pad $\left(r=R_{p}, z=0\right.$, $\theta=0.5 \theta_{0}$ and $\theta=-0.5 \theta_{0}$ are smooth (Fig. 4). At the beginning of the process the temperature increases, attaines the maxiumum value and decreases until the moment of standstill. However the temperature differences for variable angle are sllight, it may be observed that the lowest temperature is reached for the angle of the pad of $160^{\circ}$.

It should be noted that the braking process for each angle is consistent, namely the friction force is the same irrespective of the angle $\theta_{0}$. Thus the temperature calculated may be confronted and compared indicating the best geometrical configuration. Obviously when $\theta_{0}$ is greater the force possible to apply would be higher. 


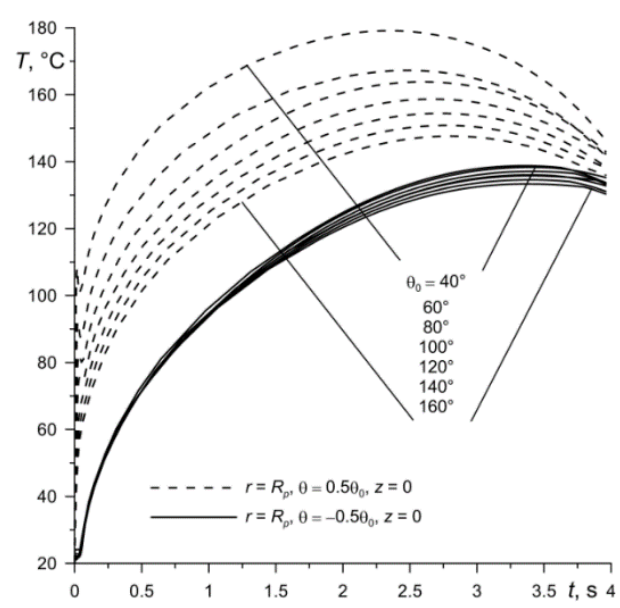

Fig. 4. Temperature evolutions on the contact surface of the pad $r=R_{p}, z=0$ at $\theta=0.5 \theta_{0}$ and $\theta=-0.5 \theta_{0}$

The presented in Fig. 4 evolutions for two point locations on the outer edge of the pad show the amplitude of oscilations of temperature of the disc presented in Fig. 5. Unlike the contact temperature of the pad, in Fig. 5 we can see the periods of frictional heating and dissipation of heat through conduction and convection to the surrounding air. An interesting is the fact that the minimum value of the oscilations through the enitre process is almost equal for each angle of the pad, wheras the maxiumum temperature is clearly higher for smaller cover angle of the pad. This relationship speaks for using greater angle of the pad.

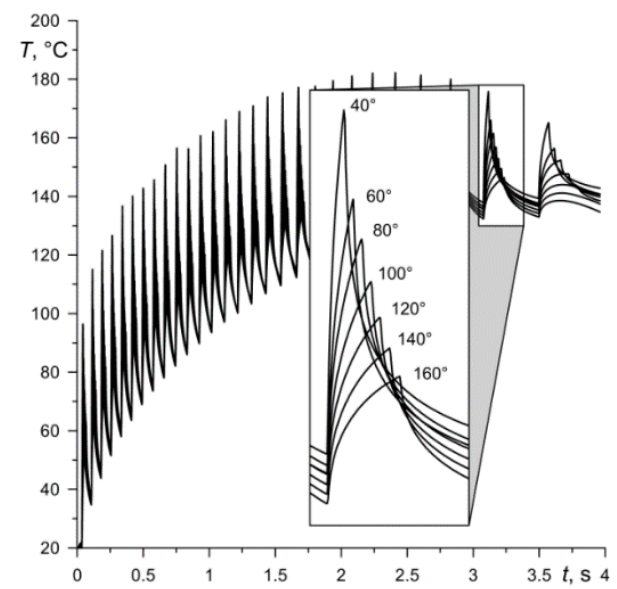

Fig. 5. Temperature evolutions on the contact surface of the disc $\left(r=R_{d}\right)$ at different cover angles of the pad $\theta_{0}$

\section{CONCLUSIONS}

In this paper temperature fields of the components of the disc brake at different cover angle of the pad $\theta_{0}$ were studied. Varying the contact pressure, for each $\theta_{0}$ the friction force was equal in order to maintain the work done also equal. Based on the obtained temperature distributions and their changes during the single braking, the following conclusions were drawn:

- the maximum temperature of the pad-disc interface is reached for the smallest $\theta_{0}$

- the difference between the maximum temperatures for the corresponding points in braking time at different cover angles of the pad is slight; the lowest difference occurs at the moment of standstill (Figs. 4, 5);
- for each studied cover angle of the pad the maximum temperature of the disc at the end of braking was approximately equal (Fig. 5).

The obtained results show that there is a clear relationship between the maximum temperature and the cover angle of the pad. However it is known, that typically the pad does not cover the rubbing path of the disc entirely. This may result from more complex caliper geometry necessary to approve uniform pressure distribution on the contact surface when increasing the $\theta_{0}$.

\section{REFERENCES}

1. Adamowicz A., Grzes P. (2011a), Analysis of disc brake temperature distribution during single braking under nonaxisymmetric load, Applied Thermal Engineering, Vol. 31, No. 6-7, 1003-1012.

2. Adamowicz A., Grzes P. (2011b), Influence of convective cooling on a disc brake temperature distribution during repetitive braking, Applied Thermal Engineering, Vol. 31, №. 14-15, 2177-2185.

3. Baranowski P., Damaziak K., Małachowski J. (2013) Brake system studies using numerical methods, Maintenance and Reliability, Vol. 15, No. 4, 337-342.

4. Baranowski P., Damziak K., Malachowski J., Mazurkiewicz L., Kastek M., Piatkowski T., Polakowski H. (2011), Experimental and numerical tests of thermo-mechanical processes occurring on brake pad lining surfaces, Surface Effects and Contact Mechanics X, Computational Methods and Experiments, Edited By: J.T.M. DE HOSSON, University of Groningen, Netherlands and C.A. Brebbia, Wessex Institute of Technology, UK, Vol. 71, 15-24.

5. Chichinadze A. V., Matveevski R.M., Braun E. P. (1986), Materials in tribotechnics non-stationary processes, Nauka, Moscow (in Russian).

6. COMSOL Multiphysics 4.4, Heat Transfer Module User's Guide, 1998-2013.

7. Ghadimi B., Kowsary F., Khorami M. (2013a) Thermal analysis of locomotive wheel-mounted brake disc, Applied Thermal Engineering, Vol. 51, №. 1-2, 948-952.

8. Ghadimi B., Sajedi R., Kowsary F. (2013b) 3D investigation of thermal stresses in a locomotive ventilated brake disc based on a conjugate thermo-fluid coupling boundary conditions, International Communications in Heat and Mass Transfer, Vol. 49, 104-109.

9. Ścieszka S. F. (1998) Friction brakes - material, structural and tribological problems, ITE, Radom (in Polish).

10. Scieszka S. F., Zolnierz M. (2014), Experimental and numerical investigations of thermo-mechanical instability of the industrial disc brakes, Proceedings of the Institution of Mechanical Engineers, Part $\mathrm{J}$ : Journal of Engineering Tribology, Vol. 228, No. 5, 567-576.

11. Talati F., Jalalifar S. (2008), Investigation of heat transfer phenomena in a ventilated disk brake rotor with straight radial rounded vanes, Journal of Applied Sciences, Vol. 8, No. 20, 35833592.

12. Talati F., Jalalifar S. (2009), Analysis of heat conduction in a disk brake system, Heat and Mass Transfer, Vol. 45, 1047-1059.

13. Yevtushenko A.A., Grzes P. (2010), The FEM-modeling of the frictional heating phenomenon in the pad/disc tribosystem (a review). Numerical Heat Transfer Part A-Applications, Vol. 58, 207-226.

14. Yevtushenko A.A., Grzes P. (2014) Mutual influence of the velocity and temperature in the axisymmetric FE model of a disc brake, International Communications in Heat and Mass Transfer, Vol. 57, 341-346.

The work has been accomplished under the research project No. MB/WM/8/2014 at the Bialystok University of Technology. 